\title{
Combining Natural Reaction Analogues and First Derivative Spectrophotometric Method to Enhance the Visible Spectra of a Non-Polar Crude Leaf Extract
}

\author{
Kwabena J. Sarfo ${ }^{1 *}$ \\ ${ }^{1}$ Department of Biochemistry, University of Cape Coast, Cape Coast, Ghana.
}

Research Article

Received $13^{\text {th }}$ November 2011

Accepted 26 ${ }^{\text {th }}$ December 2011

Online Ready $5^{\text {th }}$ March 2012

\section{ABSTRACT}

Aim: This study was aimed at using natural reaction analogues and first derivative spectrophotometry to enhance the visible range of the non polar leaf extract.

Study Design: The leaf of Alchornea cordiflora was extracted so as to enrich the extract with chlorophyll holochromes and carotenoids. The ethanolic extract was designated as MX and used as the non polar leaf extract in the experiment.

Place and Duration of Study: Department of Biochemistry, University of Cape Coast, Ghana, between March 2011 and August 2011.

Methodology: A non-polar crude leaf extract was treated with naturally occurring reaction analogues of acidification, alkalinisation, oxidation and reduction. The spectra of the resulting solutions were analyzed by difference and first derivative spectrophotometric methods.

Results: The treatment led to the enhancement of the chromophoric content and the general quality of the individual spectrum. The wavelength regions: $420-500,440-500,460-$ $500,500-600 \mathrm{~nm}$ of spectral improvement were selective to their respective analogue test substance and conditions used in a reaction

Conclusion: The 500-600 nm range was better than the Soret and the red regions to monitor the copper complexation with acidic non-polar leaf extracts of Alchornea cordiflora. Alkalinization, oxidation and reduction conditions also resolved the multiple peaks of the Soret region into distinct peaks that can be useful for monitoring and quantification of bioinorganic preparations from the leaf extract of Alchornea cordiflora.

\footnotetext{
${ }^{*}$ Corresponding author: Email: sarfojsk@yahoo.com;
} 
Keywords: Visible spectrum; Alchornea cordifolia; derivative spectrophotometric; naturally occurring reaction analogues; non-polar leaf extract.

\section{INTRODUCTION}

The current drive towards the use of plant crude extracts as against pure synthetic ligands for the synthesis of inorganic nanostructures is purely borne out of health and environmental concerns (Leela and Vivekanandan, 2008). In addition, coordination compounds with mixed ligands are more versatile than mono-liganded complexes in organic reactions (Thahira et al., 2007). The vast diversity of metabolites in plants makes plant crude extracts attractive reagents for the preparation of bioinorganic compounds with mixed activities and for environmentally benign and biocompatible nanostructures (Leela and Vivekanandan, 2008; Govindarazu et al., 2010). The use of plant extracts for the synthesis of bio-inspired nanostructures will require simple, cost effective but robust means of monitoring in order to understand the mechanism and kinetics of the reaction to ensure less defective but more homogenous chemical composition of the product. UV-visible spectrophotometry has been the method of choice of routine comparative monitoring the synthesis of chemical compounds (Silverstein et al., 1991). It is fast, simple, easy to assemble and applicable to wide range of compounds and conditions. The conventional methods of UV-visible monitoring in synthesis rely on spectral changes of either the product as it is formed or the reactants as they are consumed. The two approaches are daunting in inorganic synthesis involving non-polar leaf extracts in view of their complex spectra. The visible spectrum of a non-polar leaf extract is characterized by the superposition of chlorophylls and carotenoids peaks in the Soret region and the overwhelming broad chlorophyll peaks in the red region (Croce et al., 2001). This study was therefore aimed at evaluating the combined effects of natural reaction analogues and first derivative spectrophotometric method (Dere et al., 1998) on the enhancement of the visible spectrum of a crude non-polar leaf extract of Alchornea cordifolia that could be useful for monitoring bioinorganic synthesis. Alchornea cordifolia grows in the wild in the sub-Saharan Africa countries. The leaf extract of this plant is used in traditional medicine as anti-inflammatory, anti-bacteria, anti-fungus and antiparasitic (Mavar et. al., 2004). In Ghana the leaf extract is also used for food coloration and dyeing of cloths. In addition to pigments such as chlorophyll and carotenoids, the leaf also contains chemicals such as tannins, phenolic acids, flavonoids, and alkaloids (Mavar et. al., 2004). Improved method of monitoring the synthesis of bioinorganic compounds using the leaf extract of Alchornea cordifolia will expand the use of the plant and orientate research on its compositional standardization, and formulation for a successful commercial exploitation. For the above reasons the plant was used in this study as a model for medicinal leaf extracts and also to access its potential use in bioinorganic preparations.

\section{MATERIALS AND METHODS}

\subsection{Collection, Preparation of Non-Polar Extract, MX}

The leaf of Alchornea cordifolia was selected for this study since it is widely used in Ghana for food coloration and general dyeing purposes and also for its easiness of organic extraction. The leaves were collected and identified by the plant taxonomist at the University of Cape Coast, Ghana. The leaves were kept at $-20{ }^{\circ} \mathrm{C}$ prior to extraction after a brief washing in cold running water. $100 \mathrm{gm}$ of thawed leaves were extracted overnight by 
steeping the leaves in $1000 \mathrm{ml}$ of chilled $100 \%$ acetone. Acetone was removed by rotary evaporation and the remaining green turbid residue was diluted up to $100 \mathrm{ml}$ with distilled water and kept at $4{ }^{\circ} \mathrm{C}$ as the bulk acetone fraction prior to use. The $100 \mathrm{ml}$ bulk acetone fraction was extracted three times with $100 \mathrm{ml}$ of $100 \%$ hexane for each run. After evaporation of hexane the residues were combined and dissolved in $20 \mathrm{ml}$ of absolute ethanol and used as the non-polar crude leaf extract MX for visible spectrophotometric analysis.

\subsection{Analogue Reactions}

The analogues of the naturally occurring reactions, 1-5 were performed by treating the reagent $\mathrm{MX}$ with the appropriate analogue test substance. Different compositions of ethanol-aqueous solvent system were tested for each analogue reaction to ensure proper mixing of the non-polar components of MX and the inorganic text samples. Inappropriate combination of the two solvents could result in precipitation of a turbid MX solution. The following media containing the various analogue test samples (underlined) were set for the analogue reactions (1-5):

1. $1800 \mu \mathrm{L}$ ethanol, $0.01 \mathrm{M} \mathrm{HCl}, \mathrm{pH} 2.2$

2. $1800 \mu \mathrm{L}$ ethanol, $0.02 \mathrm{M} \mathrm{NaOH}, \mathrm{pH} 9.0$

3. $2100 \mu \mathrm{L}$ ethanol, $850 \mu \mathrm{L}$ water, $0.3 \% \underline{\mathrm{H}}_{2} \underline{\mathrm{O}}_{2}$

4. $1500 \mu \mathrm{L}$ ethanol, $700 \mu \mathrm{L}$ water, $0.04 \mathrm{M} \mathrm{NaBH}_{4}$

5. $1700 \mu \mathrm{L}$ ethanol, $1200 \mu \mathrm{L}$ of $0.1 \mathrm{M} \mathrm{HCl}, 20 \mu \mathrm{M} \mathrm{CuSO}_{4}$

The analogue test substances were prepared with double distilled water. Ethanol, acetone and hexane were analytical grade.

\subsection{Spectrophotometric Analysis}

All spectrophotometric readings were recorded with visible spectrophotometer (Jenway 6100 ) equipped with a $1-\mathrm{cm}$ light path length. For each test, absorption data were recorded for sample within the wavelength range of 400 to $700 \mathrm{~nm}$ at $10 \mathrm{~nm}$ wavelength intervals. $200 \mu \mathrm{L}$ of reagent MX was equilibrated in each of the above reaction media at $30{ }^{\circ} \mathrm{C}$ for 10 minutes for completion of reaction before absorbance was recorded with borosilicate cuvette for a final reaction volume of $3.0 \mathrm{ml}$. Spectra for the control samples of reagent MX were recorded in reaction media without the analog test substances. The media 1-5 were used as blanks and subtracted from their respective spectra. First derivative spectra were obtained by mathematically manipulating the output data of the zero order absorption spectra on spreadsheets using Microsoft Excel 2007 program.

\section{RESULTS AND DISCUSSION}

Many of the reported use of plant-extracted solutions for the preparation of bioinorganic substances have been performed in aqueous systems where metal salts and the extracted solutions are soluble. For plant aqueous extracts, polyols and water-soluble heterocyclics are the major components capable of reducing metal centers (Chobot and Hadacek, 2010). This implies that a large proportion of plant metabolites made up of non- polar molecules have not been explored for the synthesis of bioinorganic substances. The problem associated with the use of non-polar leaf extracts in bioinorganic synthesis is their limited solubility in aqueous systems where most metal salts are soluble. The complex spectrum of 
a non-polar leaf extract also makes it difficult to identify reactant (leaf extract) or product signal that can be used for monitoring the reaction. The solutions to these problems are highlighted in the sections 3.1 to 3.4. The use of hexane leaf extract obtained from the bulk acetone leaf extract, as against the direct use of hexane or acetone leaf extract for the analyses was aimed at enriching the hexane leaf extract with chlorophyll holochromes and carotenoids and also to prevent turbidity of MX treatments. MX treatments produced clear solutions in 50 to $70 \%$ ethanol in the stated combinations as shown in Section 2.2 with the analogue test substances and copper sulfate. Acid-base and redox reactions were explored in this study as the naturally occurring reaction analogues since they are the commonest physiological reactions of the cell. Copper complexation with the non-polar leaf extract was used to test the two main goals of this study. First, the mixing of the inorganic substances and the non-polar extracts at microscale level to give a clear solution with stable color suitable for spectrophotometric analysis and second, the generation of an enhanced reagent's (leaf extract) peak distinctive enough to be used for monitoring the synthesis of bioinorganics. The spectral analysis of the products of the reactions 1 to 5 is presented as Figures 1 to 5 respectively. A and B parts of each figure are the zero and first derivative spectral scans of each product. The aim is to reveal the extent of change and chromophoric content of each wavelength window of analysis and the selectivity of the change to the analogue reaction.

\subsection{Spectral Analysis of MX in Acidifying Condition}

In Figure 1, there was a clear enhancement of absorption bands in the wavelength range of 500-600 nm after acid treatment. The three overlapping derivative peaks of the control (A) sample were resolved after acid treatment (B) providing more spectral details within this window. The absorbance of a major peak around $535 \mathrm{~nm}$ was lower under acid treatment but the general spectral quality was improved with the treatment. The wavelength range 500 to $600 \mathrm{~nm}$ is transparent to pure chlorophyll and carotenoid mixtures and in the case of crude green leaf extracts can only be seen with chlorophyll band tailings that may constitute about $10-20 \%$ of the maximum chlorophyll peak (Croce et al., 2001). The region can serve as the most reliable monitor for metal complexes of plant-extracted ligands compared to the complex Soret region which was not affected by the acid treatment. Thus in addition to the general effect of $\mathrm{pH}$ changes on absorption the treatment of extracts with hydrochloric acid must have led to the accumulation of ligands including the pheophytins (Nelson and Feruzzi, 2008) which resulted in the general increase in the chromophoric content of the zero and first derivative absorption spectra in the 500 to $600 \mathrm{~nm}$ range as shown in Figure 1.

\subsection{Spectral Analysis of MX in Alkaline Medium}

In Figure 2, the first order spectra of the untreated $(A)$ and treated $(B)$ samples of reagent $M X$ were more informative than the zero order spectra. A shift of the derivative signal in the range of $420-440 \mathrm{~nm}$ without a corresponding shift in the adjacent signal after alkali treatment may be due to the different origins of the two adjacent signals in the control spectra (A). The alkali treatment did not affect the yellow-green $(500-600 \mathrm{~nm})$ and the red $(600-700)$ regions. The Soret region $(350-450 \mathrm{~nm})$ is composed of chlorophyll and carotenoid peaks and therefore the affected peak could be as a result of the saponification of the carotenoid components. 
This implies that the unaffected adjacent derivative signals in the $450 \mathrm{~nm}$ to $500 \mathrm{~nm}$ range could be due to the chlorophyll molecules since these could not have been deesterified by the mild sodium ethoxidic condition of the analogue reaction (2) (Fernandez et al., 2000).

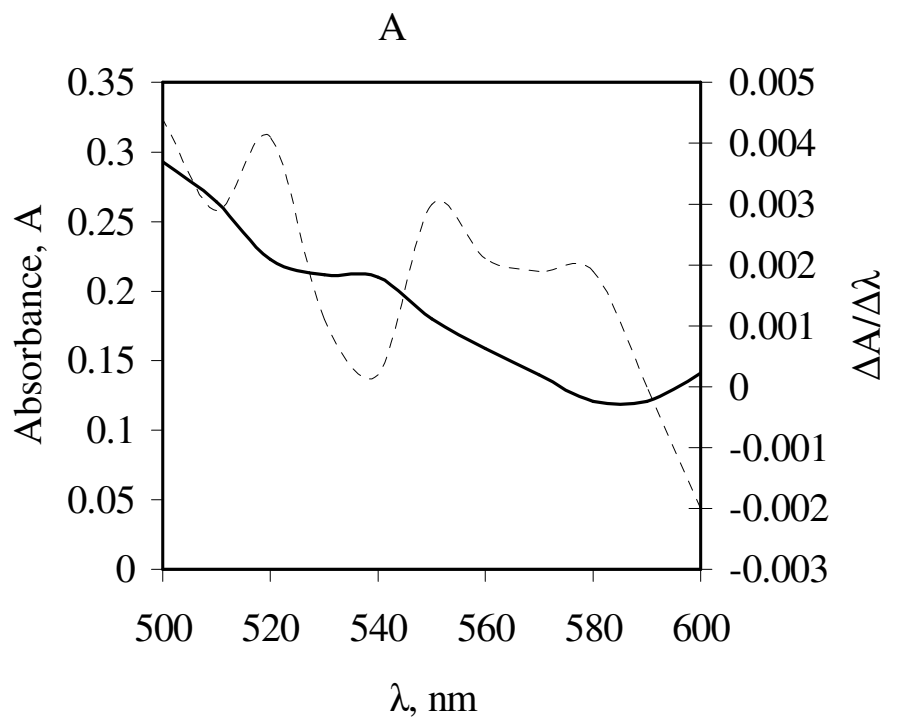

B

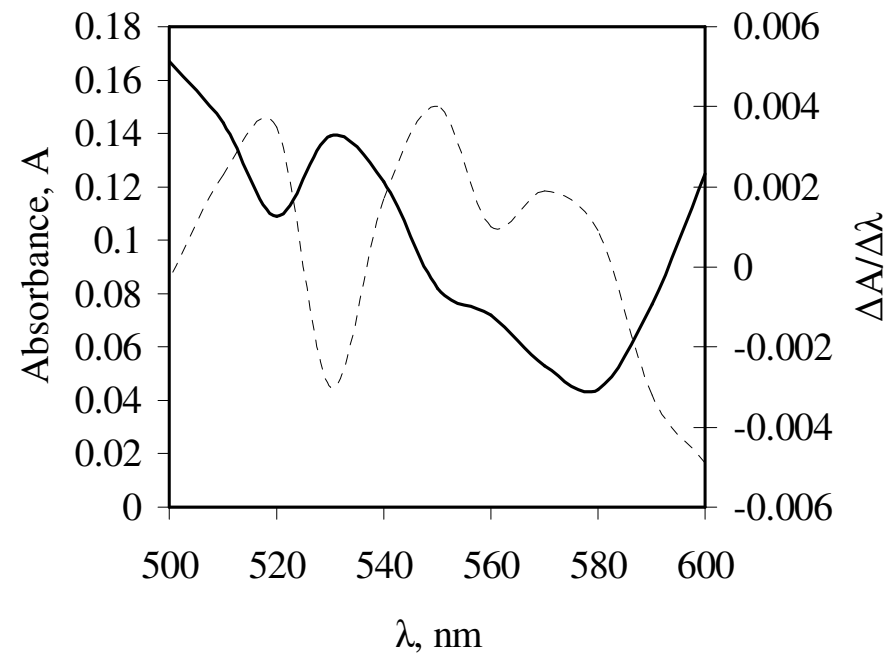

Figure 1. Visible scanning of leaf extract $M X$, in neutral $(A)$ and acidic (B) media. The acidic medium contained $0.01 \mathrm{M} \mathrm{HCl}$ at pH 2.2 Zero order (—) and first order (.....) derivatives were determined for absorbance values recorded within the wavelength range 500 to 600 

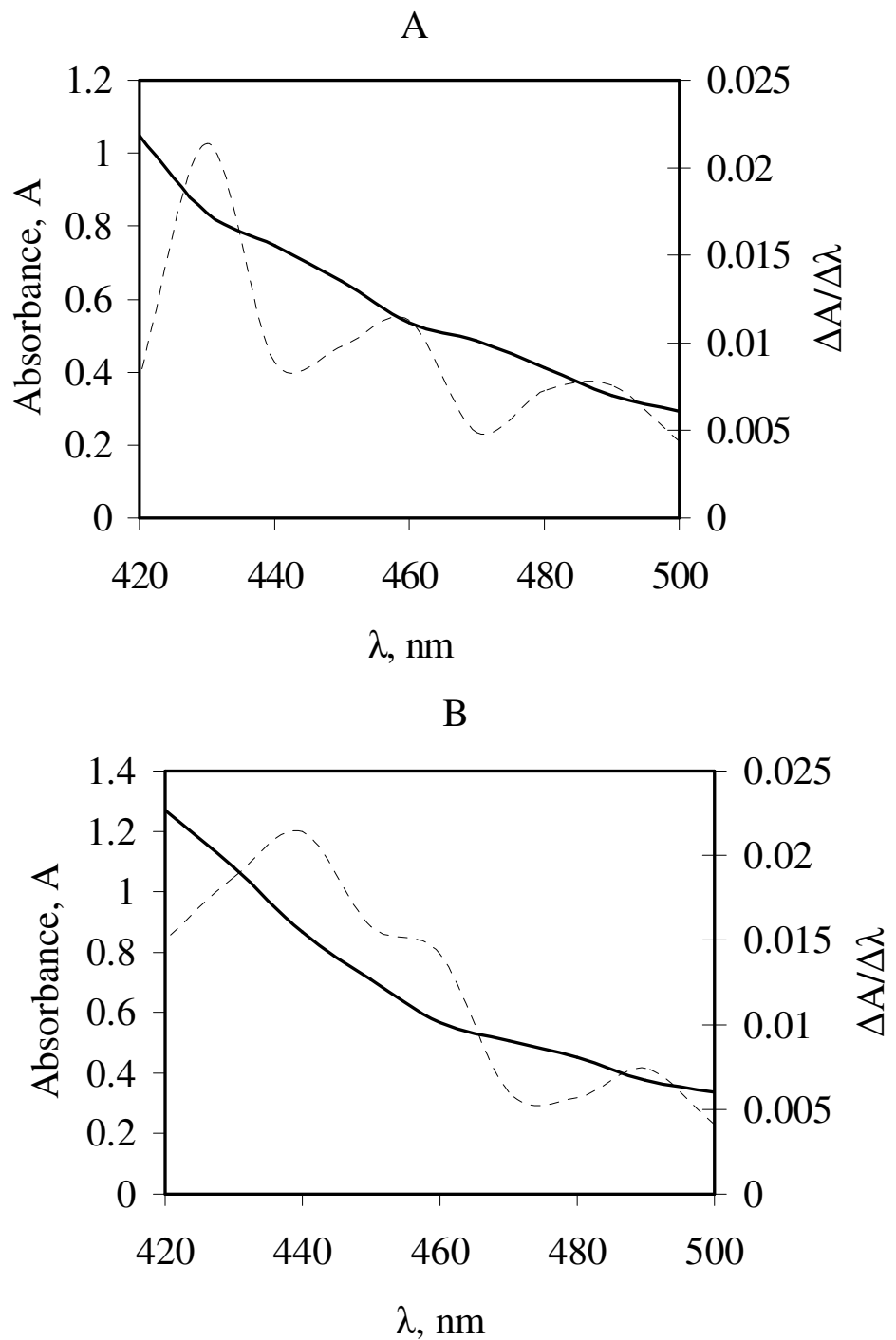

Figure 2. Visible scanning of leaf extract $M X$, in neutral (A) and alkaline (B) conditions.

The alkaline medium contained $0.02 \mathrm{M} \mathrm{NaOH}$ at $\mathrm{pH}$ 9.0. Zero order ( $\longrightarrow$ ) and first order (......) derivatives were determined for absorbance values recorded within the wavelength range 420 to 500 $n m$

\subsection{Spectral Analysis of MX in Oxidizing Medium}

In the 440-500 nm spectral window of Figure 3, the effect of oxidation on MX was not visible in the normal spectra of the treated and untreated MX. However, the first derivatives of the two spectra displayed shifting and quenching of signals resulting in a single derivative signal in this window after treatment. Hydrogen peroxide has been shown to oxidize both chlorophyll and carotenoid molecules with or without catalysis (Fenton or peroxidase) 
(Takahama et al., 2007). The first derivative peak around $455 \mathrm{~nm}$ (Figure 3B) is a sharp contrast to the corresponding zero and first order spectra of Figure $3 \mathrm{~A}$.

A

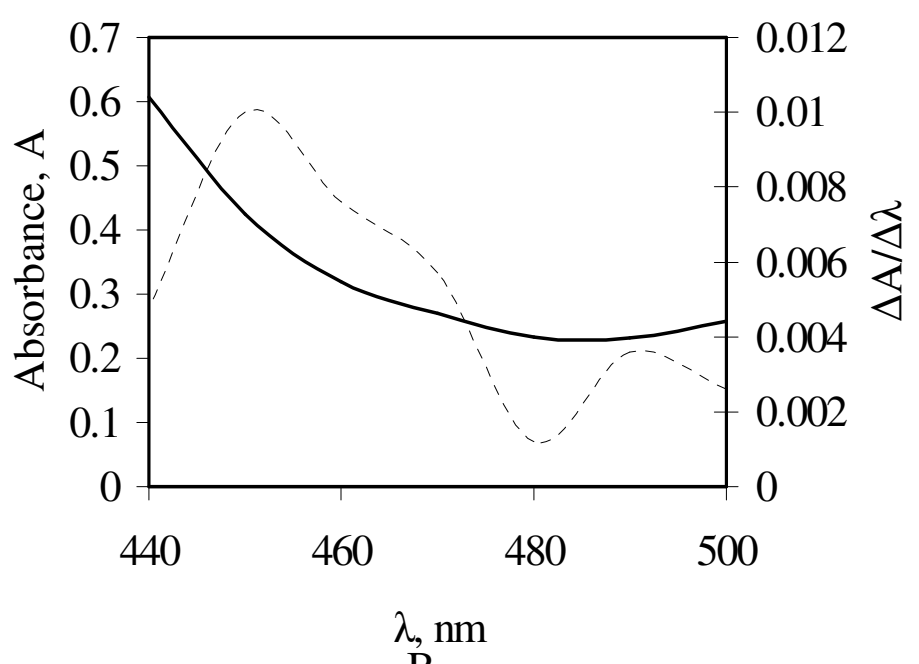

$\mathrm{B}$

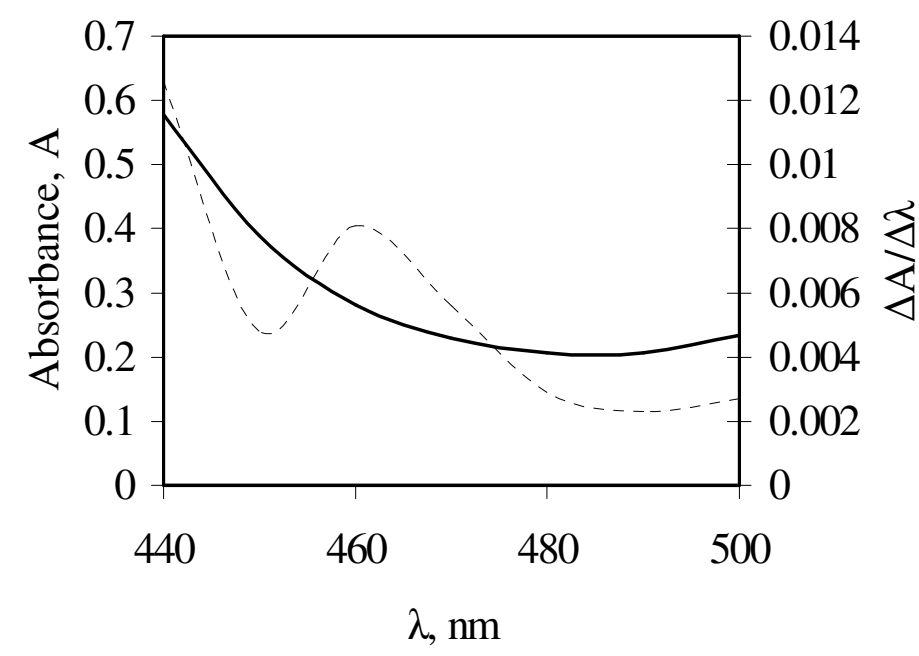

Figure 3. Visible scanning of leaf extract $M X$, in control $(A)$ and oxidizing (B) conditions.

The oxidative agent was $0.3 \% \mathrm{H}_{2} \mathrm{O}_{2}$. Zero order absorption spectrum ( $\longrightarrow$ ) was converted to first order spectrum (.....) for the wavelength range 440 to $500 \mathrm{~nm}$

\subsection{Spectral Analysis of MX in Reducing Medium}

In Figure 4, the first derivative spectrum of the $\mathrm{NaBH}_{4}$-treated sample gave around $25 \%$ increase in signal height. The symmetrical nature of this signal with an increase in depth of the adjacent valleys might indicate a unique chromogenic reaction product responsible for the observed transformation. The first derivative pattern observed in Figure 4 was similar to Figure 3 . However, the reduction gave a maximum derivative peak around $475 \mathrm{~nm}$ as 
shown in Figure 4B. Sodium borohydride has opposite spectral effect on chlorophylls and carotenoids causing red shift in chlorophylls and blue shift in carotenoids. However, it has been found that compared to chlorophylls, carotenoids are in general quite resistant to reduction. A dilute solution of sodium borohydride can readily reduce the formyl group in chlorophyll $b$ to its hydroxymethyl derivative while esters, vinyl and carboxyl groups of carotenoids are only reduced with difficulty (Chadwick et al., 1987). Thus, the unique first derivative peak maximals of the treated MX shown in Figures 3 and 4 could be due to changes in the chlorophyll pigments and not in the carotenoid molecules.

A

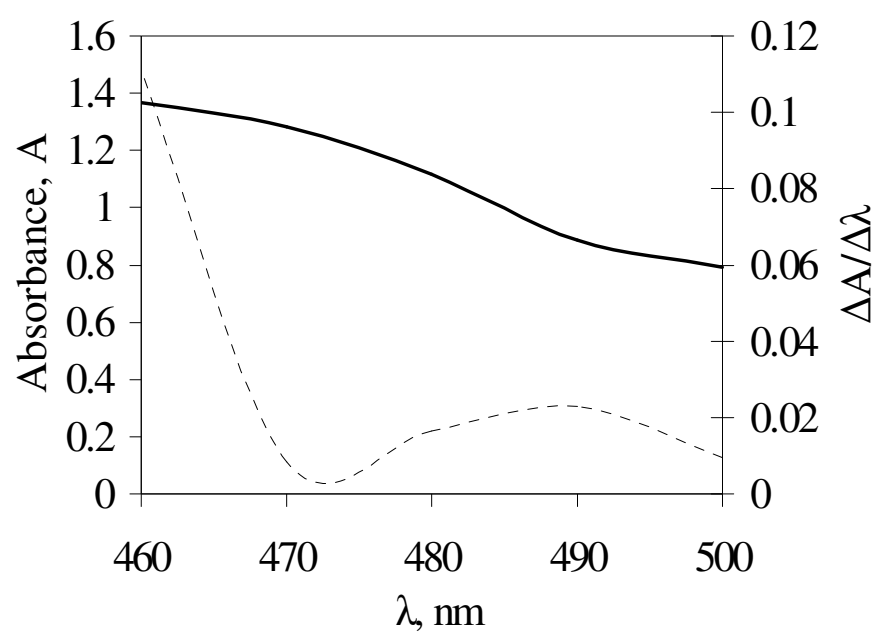

B

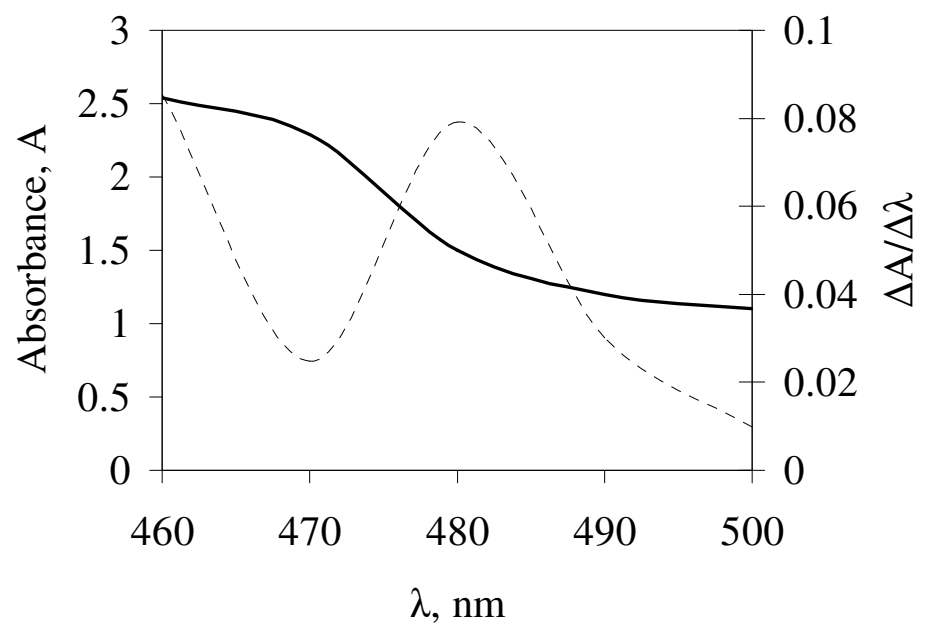

Figure 4: Visible scanning of leaf extract $M X$, in control $(A)$ and reducing $(B)$ conditions

The reductive agent was $0.04 \mathrm{M} \mathrm{NaBH}_{4}$. Zero order absorption spectrum ( $\longrightarrow$ ) was converted to first order spectrum (......) for the wavelength range 460 to $500 \mathrm{~nm}$ 


\subsection{MX-Copper Complexation Observed by Enhanced Spectrum}

In Figure 5, the significance of the unique extraction protocol, the selectivity and sensitivity of the naturally occurring reaction analogs and first derivative spectroscopy to spectral enhancement were tested by experiment reported by Nelson and Feruzzi, (2008) showing the replacement of magnesium in chlorophyll by iron in acidic condition with modifications.

A

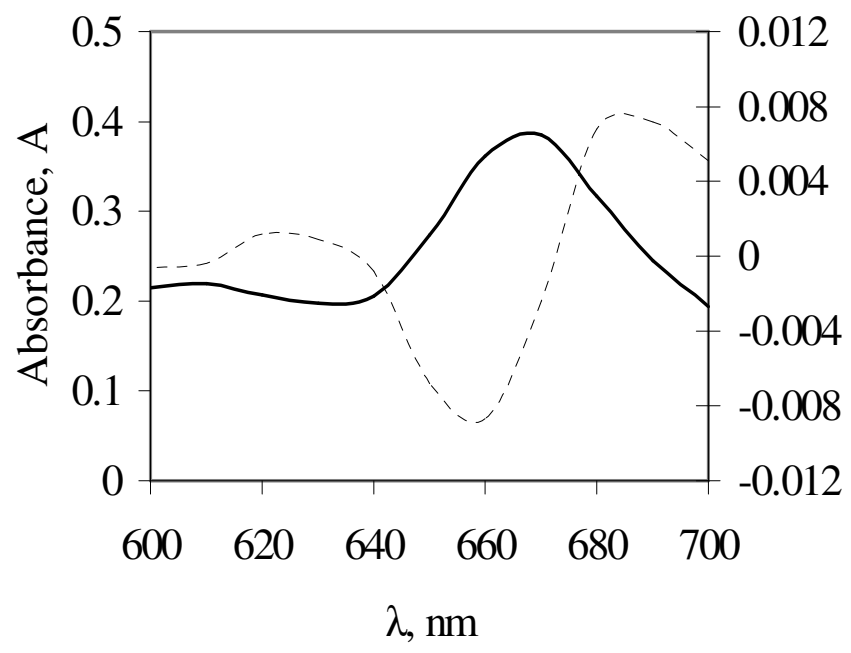

B

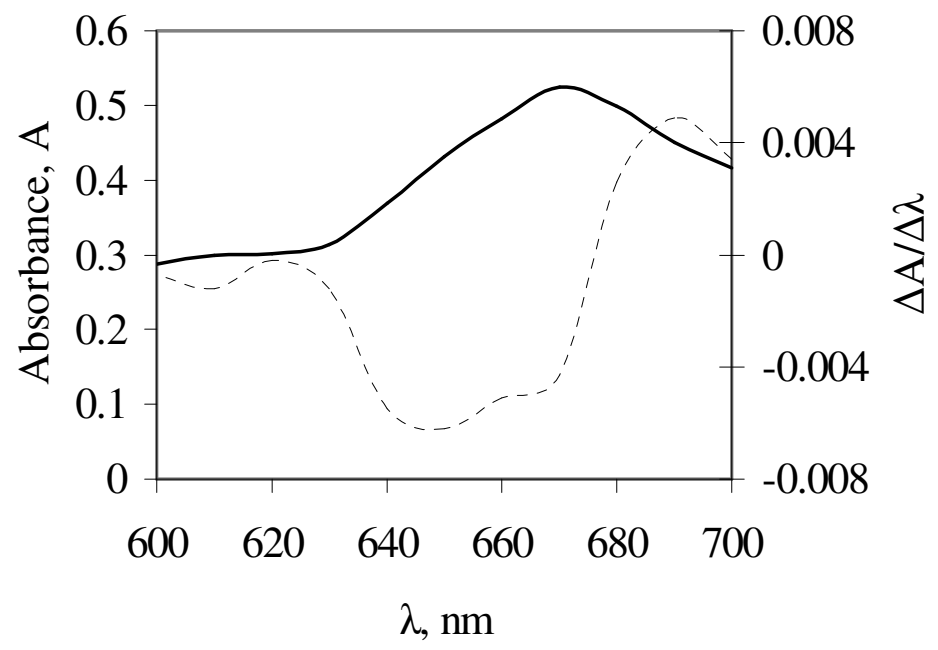

Figure 5: Visible scanning of leaf extract $M X$, in control (A) and copper complexing (B) conditions.

Copper-complexing medium contained $20 \mu \mathrm{M}$ CuSO4 in $\mathrm{HCl}$ solution. Zero order absorption spectrum (—) was converted to first order spectrum (......) for the wavelength range 600 to $700 \mathrm{~nm}$

The treatment of acidic ethanolic solution of leaf extract MX, with copper sulfate resulted in the replacement of $\mathrm{Mg}$ (II) ions by $\mathrm{Cu}$ (II) ions in chlorophyll molecules as shown by the 
splitting of a single minimum of Figure $5 \mathrm{~A}$ into two minima in Figure $5 \mathrm{~B}$. The occurrence of the acid modified $\mathrm{MgChl}$ (pheophytin, $\mathrm{H}_{2} \mathrm{Chl}$ ) peak at $\lambda_{\max }$ around $675 \mathrm{~nm}$ and the more stable CuChl peak at $\lambda_{\max }$ around $655 \mathrm{~nm}$ was better observed with first derivative spectrum than by the zero order spectrum as shown in Figure 5B. In Figure 6A the enhancement of the chromophoric content in the $500-600 \mathrm{~nm}$ range of Figure 1 was tested as the reagent's (leaf extract) own signal that can possibly monitor the accumlation of Cu-pheophytin complex product of Figure 5. As shown in Figure 6B, treatment of the acidic MX with copper sulfate resulted in a single symmetrical derivative peak in this wavelength window making this window a plausible reagent's (leaf extract) own signal that could be used to monitor Mg (II) titration with $\mathrm{Cu}(\mathrm{II})$ in chlorophyll molecules in MX as shown in Figure 5B.

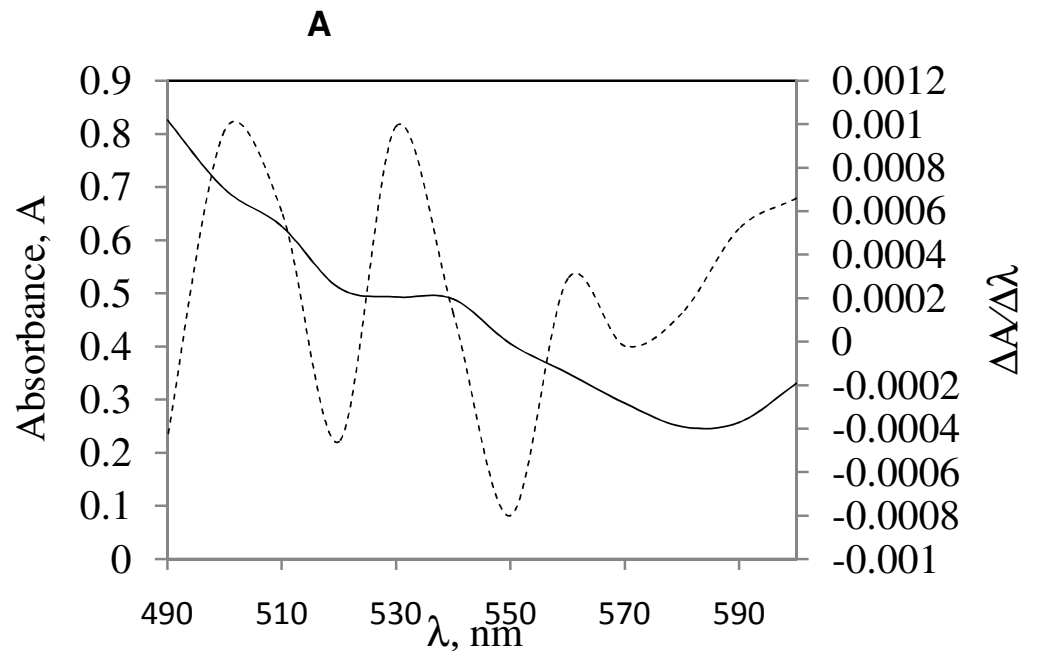

B

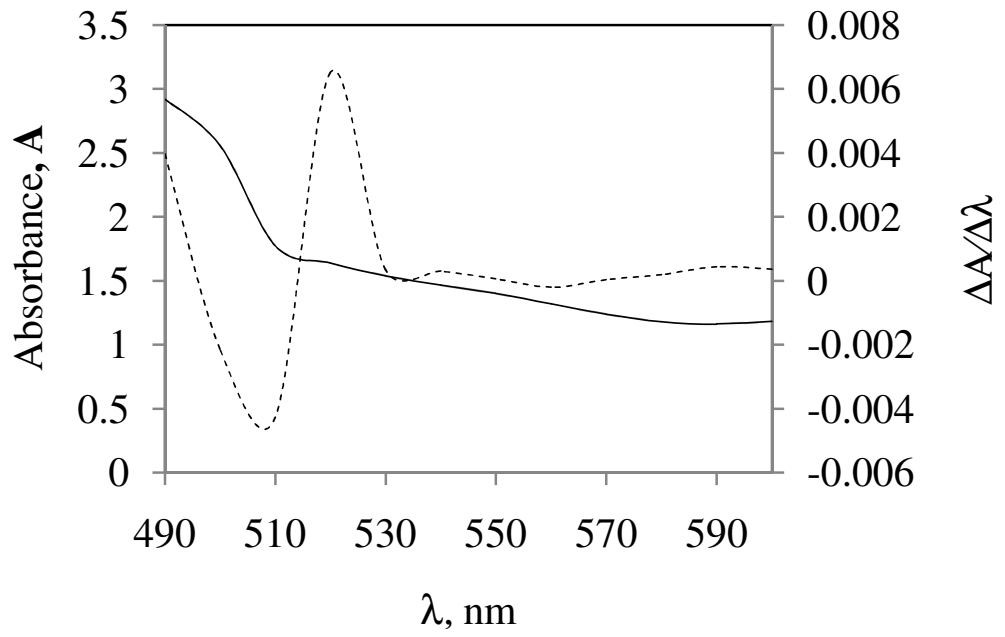

Figure 6: Visible scanning of leaf extract $M X$, in control $(A)$ and copper complexing (B) conditions.

Copper-complexing medium contained $20 \mu \mathrm{M}$ CuSO4 in $\mathrm{HCl}$ solution. Zero order absorption spectrum (—) was converted to first order spectrum (.....) for the wavelength range 500 to $600 \mathrm{~nm}$ 


\section{CONCLUSION}

Many traditional preparations obtained from food and medicinal plants owe their efficiency on the multi-component nature of their crude rather than on any purified compound (Chatterjee et al., 2010). Consequently a number of broad-based chemical analysis and applications of their crude extracts have been reported (Chatterjee et al., 2010). Bio-inspired inorganic structures are usually prepared with the crude extract of the bio-material and analyzed without resort to purifications. Spectrophotometric analysis of crude non-polar leaf extracts or their inorganic derivatives are quiet daunting in view of their complex components. In this study chemical and first derivative treatments achieved enhanced and stable spectral signals in the Soret, the uninformative yellow-green $(500-600 \mathrm{~nm})$ and the red spectral regions of a non-polar leaf extract. The improved chromophoric content has been shown as possible monitor for copper pheophytin complexation. Alkalinity, oxidative and reductive reactions resulted in specific chemical changes that gave unique absorption peaks which were treated with first derivative to give enhanced spectra in the windows 420 $500 \mathrm{~nm} ; 440-500 \mathrm{~nm}$ and $460-500 \mathrm{~nm}$ respectively. The approach could complement the use of color visualization, increasing absorbance and shift in wavelengths to monitor the synthesis of bioinorganic substances.

\section{ACKNOWLEDGEMENTS}

I am grateful to the technicians in the Department of Molecular Biology and Biotechnology, University of Cape Coast for their invaluable assistance in diverse ways.

\section{COMPETING INTERESTS}

Authors have declared that no competing interests exist.

\section{REFERENCES}

Chadwick, B.W., Zhang, C., Cogdell, R.J., Frank, H.A. (1987). The effects of lithium dodecyl sulfate and sodium borohydride on the absorption spectrum of the B800-850 lightharvesting protein of Rhodopseudomonas acidophila 7750. BBA-Bioenergetics, 893, 444-451.

Chatterjee, S., Srivastava, S., Khalid, A., Singh, N., Sangwan, R.S., Prakash Om., Roy, S.R., Rakesh, C.L.K. (2010). Comprehensive metabolic fingerprinting of Withania Somnifera leaf and root extracts. Phytochemistry, 71, 1085-1094

Chobot, V., Hadacek, F. (2010). Iron and its complexation by phenolic cellular metabolites. From oxidative stress to chemical weapons. Plant Signal Behav., 5, 4-8.

Croce, R., Cinque, G., Holzwarth, A.R., Bassi, R. (2000). The Soret absorption of carotenoids and chlorophylls in antenna complexes of higher plants. Photosynthesis Res., 64, 221-231.

Dere, S., Gunes, T., Sivaci, R. (1998). Spectrophotometric determination of chlorophyll, A, $\mathrm{B}$, and total carotenoid contents for some algae using different solvents. Turk. J. Botany, 22, 13-17.

Fernandez, X.E., Nathan, R., Shier, W., Watkins, B.A. (2000). Effect of alkali saponification, enzymatic hydrolysis and storage time on the total carotenoid concentration of Costa Rican crude palm oil. J. Food Compos. Anal., 13, 179-187. 
Govindarazu, K., Tamilselvan, S., Riruthiga, V., Singaravelu, G. (2010). Biogenic silver nanoparticles by solanum torvum and their promising antimicrobial activity. J. Biopest., 3, 394-399.

Leela, A., Vivekanandan M. (2008). Tapping the unexploited plant resources for the synthesis of silver nanoparticles. African J. Biotechnol, 17, 3162-3165.

Mavar-Manga, H., Brkic. D., Marie D.E.P., Quetin-Leclercq, J. (2004). In vitro antiinflammatory activity of Alchornea cordifolia (Schmach \& Thonn Mull, Arg (Euphorbiareae). J. Ethnopharmacol., 92, 209-214.

Nelson, R.E., Ferruzzi, M.G. (2008). Synthesis and bioaccessbility of Fe-pheophytin derivatives from crude spinach extract. J. Food Sci., 73, H89-91.

Silverstein, R.M., Bassler, G.C., Morsi, T.C. (1991). Ultraviolet Spectrometry. In Spectrometric Identification of Organic Compounds, Ed $5^{\text {th }}$, John Wiley and Son Inc.1991, Chapter 7, pp. 289-290.

Thahira, B., Ravoof, S.A., Croue, K.A., Ibrahim, I., Tahir, M., Crowley, A.R., Ali, M.A. (2007). Synthesis, characterization and bioactivity of mixed-ligand $\mathrm{Cu}(\mathrm{II})$ complexes containing Schiff bases derived from S-benzyldithiocarbamate and saccharinate complex containing S-benzyl-b-N-(acetylpyrid-2-yl) methylene dithiocarbamate. Polyhedron, 26, 1139-1165.

Takahama, U., Inoue, H., Nishimura, M. (1974). Oxidation-reductions between electron transfer components and hydrogen peroxide in photosystem II of chloroplast. Plant Cell Physiol, 15, 971-97.

(C) 2012 Sarfo; This is an Open Access article distributed under the terms of the Creative Commons Attribution License (http://creativecommons.org/licenses/by/3.0), which permits unrestricted use, distribution, and reproduction in any medium, provided the original work is properly cited. 